\title{
Exponential synchronization of complex networks with Markovian jump and mixed delays is
}

\author{
Yurong Liu ${ }^{\mathrm{a}, *}$, Zidong Wang ${ }^{\mathrm{b}}$, Xiaohui Liu ${ }^{\mathrm{b}}$ \\ a Department of Mathematics, Yangzhou University, Yangzhou 225002, PR China \\ ${ }^{\mathrm{b}}$ Department of Information Systems and Computing, Brunel University, Uxbridge, Middlesex, UB8 3PH, United Kingdom \\ Received 3 December 2007; accepted 21 February 2008 \\ Available online 15 March 2008 \\ Communicated by A.R. Bishop
}

\begin{abstract}
In this Letter, we investigate the exponential synchronization problem for an array of $N$ linearly coupled complex networks with Markovian jump and mixed time-delays. The complex network consists of $m$ modes and the network switches from one mode to another according to a Markovian chain with known transition probability. The mixed time-delays are composed of discrete and distributed delays, both of which are mode-dependent. The nonlinearities imbedded with the complex networks are assumed to satisfy the sector condition that is more general than the commonly used Lipschitz condition. By making use of the Kronecker product and the stochastic analysis tool, we propose a novel LyapunovKrasovskii functional suitable for handling distributed delays and then show that the addressed synchronization problem is solvable if a set of linear matrix inequalities (LMIs) are feasible. Therefore, a unified LMI approach is developed to establish sufficient conditions for the coupled complex network to be globally exponentially synchronized in the mean square. Note that the LMIs can be easily solved by using the Matlab LMI toolbox and no tuning of parameters is required. A simulation example is provided to demonstrate the usefulness of the main results obtained.

(c) 2008 Published by Elsevier B.V.
\end{abstract}

Keywords: Synchronization; Complex network; Markovian Jumping; Discrete time-delay; Distributed time-delay; Kronecker product; Linear matrix inequality

\section{Introduction}

The last decade has witnessed rapidly growing research interests on the dynamics analysis of complex networks since the pioneering work of Watts and Strogatz [1]. The main reason is twofold: (1) complex network exists in our daily life with examples including the Internet, the World Wide Web (WWW), the World Trade Web, linguistic webs and food webs, etc.; (2) dynamical behaviors of complex networks have found numerous applications in various fields such as physics, technology, and the life sciences. Synchronization has proven to be one of the most important controlling activities to excite the collective behavior of complex dynamical networks, and has received increasing research attention in, for example, the large-scale and complex networks of chaotic oscillators [2-5], the coupled systems exhibiting spatio-temporal chaos and autowaves [6,7], and the array of coupled neural networks [8-10].

On the other hand, time delays often occur in complex networks because of the limited speed of signals travelling through the links [11-14] and the frequently delayed couplings in biological neural networks, gene regulatory networks, communication

\footnotetext{
if This work was supported in part by the Biotechnology and Biological Sciences Research Council (BBSRC) of the UK under Grants BB/C506264/1 and 100/EGM17735, the Engineering and Physical Sciences Research Council (EPSRC) of the UK under Grants GR/S27658/01 and EP/C524586/1, an International Joint Project sponsored by the Royal Society of the UK, the Natural Science Foundation of Jiangsu Province of China under Grant BK2007075, the National Natural Science Foundation of China under Grant 60774073, and the Alexander von Humboldt Foundation of Germany.

* Corresponding author.

E-mail address: liuyurong@gmail.com (Y. Liu).
} 
networks and electrical power grids $[4,12,15]$. It has been well known that time delays can cause complex dynamics such as periodic or quasi-periodic motions, Hopf bifurcation and higher-dimensional chaos. According to the way it arises, the time-delay can be generally categorized as two classes: discrete and distributed. Note that continuously distributed delays have gained particular attention since a network usually has a spatial nature due to the presence of an amount of parallel pathways of a variety of axon sizes and lengths $[16,17]$. Recently, synchronization problems for various networks with discrete and/or distributed time-delays have extensively studied, see e.g. $[4,10,12,15,18,19]$ and the references cited therein.

In reality, complex networks may exhibit a special characteristic called network mode switching. For example, in [20-22], it has been revealed that a neural network sometimes has finite modes that switch from one to another at different times, and such a switching (or jumping) can be governed by a Markovian chain. In [23], the bufferless packet switching of trees and leveled networks has been illustrated to be achievable with certain network topologies. In [24], a sensor network has been shown to have jumping behavior due to the network's working environment (normal or hazardous) and the mobility of sensor node. In [25], it has been concluded that the shuffle-exchange networks can model practical interconnection systems due to their size of its switching (jumping) elements and uncomplicated configuration. In [26], the associative memory of a stochastic Hopfield-like neural automata has been reported to jump between pattern attractors. It is worth mentioning that the control and filtering problems for dynamical systems with Markovian jumping parameters have already been widely studied, see e.g. [22,27-29] and the references therein. Although complex networks with Markovian jumping parameters have great application potential in a variety of areas, there has been very little existing literature on the synchronization problem for Markovian jumping complex networks with or without mixed time-delays, and the purpose of this Letter is therefore to shorten such a gap.

In this Letter, we deal with the synchronization problem for an array of coupled complex networks with simultaneous presence of both the discrete and distributed time-delays. The addressed complex network consists of $m$ modes and the network switches from one mode to another according to a Markovian chain with known transition probability. By utilizing a novel LyapunovKrasovskii functional and the Kronecker product, we show that the addressed synchronization problem is solvable if a set of linear matrix inequalities (LMIs) are feasible. The main novelty of this Letter can be summarized as follows: (1) a new class of complex networks is proposed that contain Markovian jumping parameters; (2) both the discrete and distributed time-delays are considered that are dependent on the jumping mode; (3) rather than the commonly used Lipschitz-type function, a more general sector-like nonlinear function is employed; and (4) a new Lyapunov-Krasovskii functional is exploited to cater the mode-dependent distributed delays. A simulation example is provided to show the usefulness of the proposed global synchronization condition.

Notations. The notations are quite standard. Throughout this Letter, $\mathbb{R}^{n}$ and $\mathbb{R}^{n \times m}$ denote, respectively, the $n$-dimensional Euclidean space and the set of all $n \times m$ real matrices. The superscript " $T$ " denotes matrix transposition and the notation $X \geqslant Y$ (respectively, $X>Y$ ) where $X$ and $Y$ are symmetric matrices, means that $X-Y$ is positive semidefinite (respectively, positive definite). Let $I_{n}$ be the $n \times n$ identity matrix, and $|\cdot|$ denote the Euclidean norm in $\mathbb{R}^{n}$. If $A$ is a square matrix, denote by $\lambda_{\max }(A)$ (respectively, $\left.\lambda_{\min }(A)\right)$ means the largest (respectively, smallest) eigenvalue of $A$. The notation $A \otimes B$ stands for the Kronecker product of matrices $A$ and $B$. For $h>0, C\left([-h, 0] ; \mathbb{R}^{n}\right)$ denotes the family of continuous functions $\varphi$ from $[-h, 0]$ to $\mathbb{R}^{n}$ with the norm $\|\varphi\|=\sup _{-h \leqslant \theta \leqslant 0}|\varphi(\theta)|, l_{2}[0, \infty]$ is the space of square integrable vector. Moreover, let $\left(\boldsymbol{\Omega}, \mathcal{F},\left\{\mathcal{F}_{t}\right\}_{t} \geqslant 0, \mathcal{P}\right)$ be a complete probability space with a filtration $\left\{\mathcal{F}_{t}\right\}_{t} \geqslant 0$ satisfying the usual conditions (i.e., the filtration contains all $P$-null sets and is right continuous). Denote by $L_{\mathcal{F}_{0}}^{p}\left([-h, 0] ; \mathbb{R}^{n}\right)$ the family of all $\mathcal{F}_{0}$-measurable $C\left([-h, 0] ; \mathbb{R}^{n}\right)$-valued random variables $\xi=\{\xi(\theta):-h \leqslant \theta \leqslant 0\}$ such that $\sup _{-h \leqslant \theta \leqslant 0} \mathbb{E}|\xi(\theta)|^{p}<\infty$ where $\mathbb{E}\{\cdot\}$ stands for the mathematical expectation operator with respect to the given probability measure $\mathcal{P}$. The asterisk $*$ in a symmetric matrix is used to denote term that is induced by symmetry. Sometimes, the arguments of a function will be omitted in the analysis when no confusion can arise.

\section{Problem formulation}

Let $r(t)(t \geqslant 0)$ be a right-continuous Markovian chain on a probability space $\left(\boldsymbol{\Omega}, \mathcal{F},\left\{\mathcal{F}_{t}\right\}_{t \geqslant 0}, \mathcal{P}\right)$ taking values in a finite state space $\mathcal{N}=\{1,2, \ldots, m\}$ with generator $\Pi=\left\{\pi_{i j}\right\}$ given by

$$
P\{r(t+\Delta)=j \mid r(t)=i\}= \begin{cases}\pi_{i j} \Delta+o(\Delta), & \text { if } i \neq j, \\ 1+\pi_{i j} \Delta+o(\Delta), & \text { if } i=j .\end{cases}
$$

Here $\Delta>0$, and $\pi_{i j} \geqslant 0$ is the transition rate from $i$ to $j$ if $j \neq i$ while

$$
\pi_{i i}=-\sum_{j \neq i} \pi_{i j} .
$$

Consider the following complex dynamical network coupled by $N$ identical nodes with Markovian jumping parameters and mixed time-delays:

$$
\frac{d x_{k}(t)}{d t}=f\left(x_{k}(t), r(t)\right)+g\left(x_{k}\left(t-\tau_{1, r(t)}\right)\right)+\int_{t-\tau_{2, r(t)}}^{t} h\left(x_{k}(s)\right) d s+\sum_{l=1}^{N} w_{k l} \Gamma_{r(t)} x_{l}(t), \quad k=1,2, \ldots, N,
$$


where $x_{k}(t)=\left[x_{k 1}(t), x_{k 2}(t), \ldots, x_{k n}(t)\right]^{T}$ is the state vector of the $k$ th node; $\{r(t), t>0\}$ is the continuous-time Markov process which describes the evolution of the mode at time $t ; f\left(x_{k}(t), i\right)=\left(f_{1}\left(x_{k 1}(t), i\right), \ldots, f_{n}\left(x_{k n}(t), i\right)\right)^{T}, g\left(x_{k}\left(t-\tau_{1, i}\right)\right)=$ $\left(g_{1}\left(x_{k 1}\left(t-\tau_{1, i}\right)\right), \ldots, g_{n}\left(x_{k n}\left(t-\tau_{1, i}\right)\right)\right)^{T}, h\left(x_{k}(s)\right)=\left(h_{1}\left(x_{k 1}(s)\right), \ldots, h_{n}\left(x_{k n}(s)\right)\right)^{T}$ with $f(\cdot, i)(i=1,2, \ldots, m), g(\cdot)$ and $h(\cdot)$ being continuous nonlinear vector functions; $\tau_{1, r(t)}$ denotes the discrete time delay of the network in the mode $r(t)$, and $\tau_{2, r}(t)$ stands for the distributed time delay in the mode $r(t) ; \Gamma_{r(t)}=\operatorname{diag}\left\{\gamma_{1, r(t)}, \gamma_{2, r(t)}, \ldots, \gamma_{n, r(t)}\right\} \geqslant 0$ is a matrix linking the $l$ th state variable of each node in the network mode $r(t)$ if $\gamma_{l, r(t)} \neq 0$; and $W=\left(w_{k l}\right) \in \mathbb{R}^{N \times N}$ is the coupled configuration matrix of the network with $w_{k l} \geqslant 0(k \neq l)$ but not all zero.

For notation simplicity, we let

$$
\begin{aligned}
& x(t)=\left(x_{1}^{T}(t), x_{2}^{T}(t), \ldots, x_{N}^{T}(t)\right)^{T}, \\
& F(x(t), r(t))=\left(f^{T}\left(x_{1}(t), r(t)\right), f^{T}\left(x_{2}(t), r(t)\right), \ldots, f^{T}\left(x_{N}(t), r(t)\right)\right)^{T}, \\
& G(x(t))=\left(g^{T}\left(x_{1}(t)\right), g^{T}\left(x_{2}(t)\right), \ldots, g^{T}\left(x_{N}(t)\right)\right)^{T}, \\
& H(x(t))=\left(h^{T}\left(x_{1}(t)\right), h^{T}\left(x_{2}(t)\right), \ldots, h^{T}\left(x_{N}(t)\right)\right)^{T} .
\end{aligned}
$$

With the matrix Kronecker product, we can rewrite the network (1) in the following compact form:

$$
\frac{d x(t)}{d t}=F(x(t), r(t))+G\left(x\left(t-\tau_{1, r(t)}\right)\right)+\int_{t-\tau_{2, r(t)}}^{t} H(x(s)) d s+\left(W \otimes \Gamma_{r(t)}\right) x(t) .
$$

Remark 1. The complex network model (1) or (2) is quite general, which contains the Markovian jumping parameters and the mixed time-delays. Furthermore, the nonlinear function $F(\cdot, \cdot)$, the discrete time-delay and the distributed time delay are all dependent on the Markovian jumping modes. It should be pointed out that the synchronization problem for complex networks with both discrete and distributed time-delays has not received much attention in the literature, not to mention the case when the Markovian mode jumps are also involved.

Throughout this Letter, we make the following assumptions:

Assumption 1. (See $[12,15]$.) The coupling configuration matrix $W=\left(w_{i j}\right)$ is symmetric, i.e., $W=W^{T}$, and satisfies

$$
\sum_{l=1}^{N} w_{k l}=\sum_{l=1}^{N} w_{l k}=0, \quad k=1,2, \ldots, N .
$$

Assumption 2. (See $[16,17,30]$.) For $\forall x, y \in \mathbb{R}^{n}, i \in\{1,2, \ldots, m\}$, the nonlinear functions $f(\cdot, i), g(\cdot)$ and $h(\cdot)$ satisfy

$$
\begin{aligned}
& {\left[f(x, i)-f(y, i)-B_{1, i}(x-y)\right]^{T}\left[f(x, i)-f(y, i)-B_{2, i}(x-y)\right] \leqslant 0,} \\
& {\left[g(x)-g(y)-D_{1}(x-y)\right]^{T}\left[g(x)-g(y)-D_{2}(x-y)\right] \leqslant 0,} \\
& {\left[h(x)-h(y)-V_{1}(x-y)\right]^{T}\left[h(x)-h(y)-V_{2}(x-y)\right] \leqslant 0,}
\end{aligned}
$$

where $B_{1, i}, B_{2, i}, D_{1}, D_{2}, V_{1}, V_{2}$ are known constant matrices.

Remark 2. The conditions (4)-(6) are known as sector-like description of the nonlinearities, which are in a more general form than the usual Lipschitz functions. By adopting such a presentation, it would be possible to reduce the conservatism of the main results caused by quantifying the nonlinear functions via an LMI technique.

Definition 1. The complex network (1) or (2) is said to be globally exponentially synchronized in the mean square if, for any solution $x(t)$, there exist constants $\mu>0$ and $\alpha>0$ such that

$$
\mathbb{E}\left\{\left|x_{k}(t)-x_{l}(t)\right|^{2}\right\} \leqslant \mu e^{-\alpha t}
$$

holds for any $k, l \in\{1,2, \ldots, N\}$.

In this Letter, our main aim is to deal with the exponential mean-square synchronization problem of the network (1) or (2), and derive LMI-based sufficient conditions that guarantee the network to be exponentially synchronized in the mean square. 


\section{Main results and proofs}

Before stating our main results, we introduce the following lemmas.

Lemma 1. Let $\mathcal{U}=\left(\alpha_{i j}\right)_{N \times N}, P \in \mathbb{R}^{n \times n}, x=\left(x_{1}^{T}, x_{2}^{T}, \ldots, x_{N}^{T}\right)^{T}$ and $y=\left(y_{1}^{T}, y_{2}^{T}, \ldots, y_{N}^{T}\right)^{T}$ with $x_{k}, y_{k} \in \mathbb{R}^{n}(k=1,2, \ldots, N)$. If $\mathcal{U}=\mathcal{U}^{T}$ and each row sum of $\mathcal{U}$ is zero, then

$$
x^{T}(\mathcal{U} \otimes P) y=-\sum_{1 \leqslant i<j \leqslant N} \alpha_{i j}\left(x_{i}-x_{j}\right)^{T} P\left(y_{i}-y_{j}\right) .
$$

Lemma 2. (See [31].) Let $a<b, M \in \mathbb{R}^{n \times n}$ be a positive semi-definite matrix, and $\omega(\cdot):[a, b] \rightarrow \mathbb{R}^{n}$ be a vector function. If the integrations concerned are well defined, then the following inequality holds:

$$
\left(\int_{a}^{b} \omega(s) d s\right)^{T} M\left(\int_{a}^{b} \omega(s) d s\right) \leqslant(b-a) \int_{a}^{b} \omega^{T}(s) M \omega(s) d s .
$$

Denote

$$
\begin{array}{lcl}
\bar{\pi}=\max _{1 \leqslant i \leqslant m}\left\{\left|\pi_{i i}\right|\right\}, & \bar{\tau}_{1}=\max _{1 \leqslant i \leqslant m}\left\{\tau_{1, i}\right\}, & \bar{\tau}_{2}=\max _{1 \leqslant i \leqslant m}\left\{\tau_{2, i}\right\}, \\
\underline{\tau}_{1}=\min _{1 \leqslant i \leqslant m}\left\{\tau_{1, i}\right\}, & \underline{\tau}_{2}=\min _{1 \leqslant i \leqslant m}\left\{\tau_{2, i}\right\}, & \tau=\max \left\{\bar{\tau}_{1}, \bar{\tau}_{2}\right\} .
\end{array}
$$

We are now in a position to give the main results of this Letter as follows.

Theorem 1. Under Assumptions 1 and 2, the network (1) or (2) is globally exponentially synchronized in the mean square if there exist two positive definite matrices $Q$ and $R$, a set of positive definite matrices $P_{i}(i=1,2, \ldots, m)$ and three positive scalar constants $\delta_{1}, \delta_{2}$ and $\delta_{3}$ such that the following LMIs hold:

$$
\begin{gathered}
\Omega(k, l, i)=\left[\begin{array}{cccccc}
-N w_{k l} \hat{\Gamma}_{i}+\bar{P}_{i}+\Xi_{i} & P_{i}+\delta_{1} \breve{B}_{2, i} & \delta_{2} \breve{D}_{2} & P_{i} & \delta_{3} \breve{V}_{2} & P_{i} \\
* & -\delta_{1} I & 0 & 0 & 0 & 0 \\
* & * & c_{1} Q-\delta_{2} I & 0 & 0 & 0 \\
* & * & * & -Q & 0 & 0 \\
* & * & * & * & c_{2 i} R-\delta_{3} I & 0 \\
* & * & * & * & * & -\frac{1}{\tau_{2, i}} R
\end{array}\right]<0, \\
1 \leqslant k<l \leqslant N, \quad 1 \leqslant i \leqslant m
\end{gathered}
$$

where

$$
\begin{aligned}
& \breve{B}_{1, i}=\left(B_{1, i}^{T} B_{2, i}+B_{2, i}^{T} B_{1, i}\right) / 2 ; \quad \breve{B}_{2, i}=\left(B_{1, i}^{T}+B_{2, i}^{T}\right) / 2 ; \\
& \breve{D}_{1}=\left(D_{1}^{T} D_{2}+D_{2}^{T} D_{1}\right) / 2 ; \quad \breve{D}_{2}=\left(D_{1}^{T}+D_{2}^{T}\right) / 2 ; \\
& \breve{V}_{1}=\left(V_{1}^{T} V_{2}+V_{2}^{T} V_{1}\right) / 2 ; \quad \breve{V}_{2}=\left(V_{1}^{T}+V_{2}^{T}\right) / 2 ; \\
& c_{1}=1+\bar{\pi}\left(\bar{\tau}_{1}-\underline{\tau}_{1}\right), \quad c_{2 i}=\tau_{2, i}+\frac{1}{2} \bar{\pi}\left(\bar{\tau}_{2}^{2}-\underline{\tau}^{2}\right) ; \\
& \hat{\Gamma}_{i}=P_{i} \Gamma_{i}+\Gamma_{i} P_{i}, \quad \bar{P}_{i}=\sum_{1 \leqslant j \leqslant m} \pi_{i j} P_{j}, \quad \Xi_{i}=-\delta_{1} \breve{B}_{1, i}-\delta_{2} \breve{D}_{1}-\delta_{3} \breve{V}_{1} .
\end{aligned}
$$

Proof. Firstly, it can be inferred from (8) that there exists a constant $\vartheta>0$ such that

$$
\Omega_{\vartheta}(k, l, i):=\Omega(k, l, i)+\vartheta \operatorname{diag}\{\overbrace{1,1, \ldots, 1}^{n}, \overbrace{0,0, \ldots, 0}^{n n}\}<0 .
$$

Since $\{(x(t), r(t)), t \geqslant 0\}$ is not a Markov process, in order to cast our model into the framework for a Markov system, let us define a new Markov process $\left\{x_{t}, r(t), t \geqslant 0\right\}$ with

$$
x_{t}(s)=x(t+s), \quad-\tau \leqslant s \leqslant 0 .
$$

Consider the following stochastic Lyapunov functional candidate for the complex network (1) or (2): 


$$
\begin{aligned}
V\left(x_{t}, t, r(t)\right)= & x^{T}(t)\left(U \otimes P_{r(t)}\right) x(t)+\int_{t-\tau_{1, r(t)}}^{t} G^{T}(x(s))(U \otimes Q) G(x(s)) d s \\
& +\bar{\pi} \int_{\underline{\tau}_{1}}^{\bar{\tau}_{1}} \int_{t-s}^{t} G^{T}(x(\theta))(U \otimes Q) G(x(\theta)) d \theta d s+\int_{0}^{\tau_{2, r(t)}} \int_{t-s}^{t} H^{T}(x(\theta))(U \otimes R) H(x(\theta)) d \theta d s \\
& +\bar{\pi} \int_{\underline{\tau}_{2}}^{\bar{\tau}_{2}} \int_{0}^{u} \int_{t-s}^{t} H^{T}(x(\theta))(U \otimes R) H(x(\theta)) d \theta d s d u+\varepsilon_{0} \int_{0}^{\tau} \int_{t-s}^{t} x^{T}(\theta)\left(U \otimes I_{n}\right) x(\theta) d \theta d s,
\end{aligned}
$$

where

$$
\varepsilon_{0}=\vartheta / \tau \quad \text { and } \quad U=\left[\begin{array}{cccc}
N-1 & -1 & \cdots & -1 \\
-1 & N-1 & \cdots & -1 \\
\cdots & \cdots & \cdots & \cdots \\
-1 & -1 & \cdots & N-1
\end{array}\right]_{N \times N} .
$$

Let $\mathcal{L}$ be the weak infinitesimal generator of the random process $\left\{\left(x_{t}, r(t)\right), t \geqslant 0\right\}$ along the network (1) or (2). Then,

$$
\begin{aligned}
& \mathcal{L} V\left(x_{t}, t, i\right) \\
& =2 x^{T}(t)\left(U \otimes P_{i}\right)\left(F(x(t), i)+G\left(x\left(t-\tau_{1, i}\right)\right)+\int_{t-\tau_{2, i}}^{t} H(x(s)) d s+\left(W \otimes \Gamma_{i}\right) x(t)\right) \\
& \quad+\sum_{j=1}^{m} \pi_{i j} x^{T}(t)\left(U \otimes P_{j}\right) x(t)+G^{T}(x(t))(U \otimes Q) G(x(t))-G^{T}\left(x\left(t-\tau_{1, i}\right)\right)(U \otimes Q) G\left(x\left(t-\tau_{1, i}\right)\right) \\
& +\sum_{j=1}^{m} \pi_{i j} \int_{t-\tau_{1, j}}^{t} G^{T}(x(s))(U \otimes Q) G(x(s)) d s+\bar{\pi}\left(\bar{\tau}_{1}-\underline{\tau}_{1}\right) G^{T}(x(t))(U \otimes Q) G(x(t)) \\
& \quad-\bar{\pi} \int_{\underline{\tau}_{1}}^{\bar{\tau}_{1}} G^{T}(x(t-s))(U \otimes Q) G(x(t-s)) d s+\tau_{2, i} H^{T}(x(t))(U \otimes R) H(x(t)) \\
& \quad-\int_{0}^{\tau_{2, i}} H^{T}(x(t-s))(U \otimes R) H(x(t-s)) d s+\sum_{j=1}^{m} \pi_{i, j} \int_{0}^{\tau_{2, j}} \int_{t-s}^{t} H^{T}(x(\theta))(U \otimes R) H(x(\theta)) d \theta d s \\
& \quad+\bar{\pi} \int_{\underline{\tau}_{2}}^{\bar{\tau}_{2}} \int_{0}^{u} H^{T}(x(t))(U \otimes R) H(x(t)) d s d u-\bar{\pi} \int_{\underline{\tau}_{2}}^{u} \int_{0}^{u} H^{T}(x(t-s))(U \otimes R) H(x(t-s)) d s d u \\
& +\varepsilon_{0} \tau x^{T}(t)\left(U \otimes I_{n}\right) x(t)-\varepsilon_{0} \int_{0}^{\tau} x^{T}(t-s)\left(U \otimes I_{n}\right) x(t-s) d s .
\end{aligned}
$$

It is obvious that

$$
\begin{aligned}
& -\bar{\pi} \int_{\underline{\tau}_{1}}^{\bar{\tau}_{1}} G^{T}(x(t-s))(U \otimes Q) G(x(t-s)) d s=-\bar{\pi} \int_{t-\bar{\tau}_{1}}^{t-\underline{\tau}_{1}} G^{T}(x(s))(U \otimes Q) G(x(s)) d s, \\
& -\int_{0}^{\tau_{2, i}} H^{T}(x(t-s))(U \otimes R) H(x(t-s)) d s=-\int_{t-\tau_{2, i}}^{t} H^{T}(x(s))(U \otimes R) H(x(s)) d s, \\
& \bar{\pi} \int_{\underline{\tau_{2}}}^{\bar{\tau}_{2}} \int_{0}^{u} H^{T}(x(t))(U \otimes R) H(x(t)) d s d u=\frac{1}{2} \overline{\bar{\pi}}\left(\bar{\tau}_{2}^{2}-\underline{\tau}_{2}{ }^{2}\right) H^{T}(x(t))(U \otimes R) H(x(t)),
\end{aligned}
$$




$$
\begin{aligned}
& -\bar{\pi} \int_{\underline{\tau}_{2}}^{\bar{\tau}_{2}} \int_{0}^{u} H^{T}(x(t-s))(U \otimes R) H(x(t-s)) d s d u=-\bar{\pi} \int_{\underline{\tau}_{2}}^{\bar{\tau}_{2}} \int_{t-s}^{t} H^{T}(x(\theta))(U \otimes R) H(x(\theta)) d \theta d s, \\
& -\varepsilon_{0} \int_{0}^{\tau} x^{T}(t-s)\left(U \otimes I_{n}\right) x(t-s) d s=-\varepsilon_{0} \int_{t-\tau}^{t} x^{T}(s)\left(U \otimes I_{n}\right) x(s) d s .
\end{aligned}
$$

Also, it is not difficult to verify that

$$
\begin{aligned}
& \sum_{j=1}^{m} \pi_{i j} \int_{t-\tau_{1, j}}^{t} G^{T}(x(s))(U \otimes Q) G(x(s)) d s \\
& \quad=\sum_{j \neq i}^{m} \pi_{i j} \int_{t-\tau_{1, j}}^{t} G^{T}(x(s))(U \otimes Q) G(x(s)) d s+\pi_{i i} \int_{t-\tau_{1, i}}^{t} G^{T}(x(s))(U \otimes Q) G(x(s)) d s \\
& \quad \leqslant \sum_{j \neq i}^{m} \pi_{i j} \int_{t-\bar{\tau}_{1}}^{t} G^{T}(x(s))(U \otimes Q) G(x(s)) d s+\pi_{i i} \int_{t-\underline{\tau}_{1}}^{t} G^{T}(x(s))(U \otimes Q) G(x(s)) d s \\
& \quad=-\pi_{i i} \int_{t-\bar{\tau}_{1}}^{t} G^{T}(x(s))(U \otimes Q) G(x(s)) d s+\pi_{i i} \int_{t-\underline{\tau}_{1}}^{t} G^{T}(x(s))(U \otimes Q) G(x(s)) d s \\
& \quad \leqslant \int_{t-\underline{\tau} 1}^{T} G_{t-\bar{\tau}_{1}}^{T}(x(s))(U \otimes Q) G(x(s)) d s .
\end{aligned}
$$

Similarly, we have

$$
\sum_{j=1}^{m} \pi_{i j} \int_{0}^{\tau_{2, j}} \int_{t-s}^{t} H^{T}(x(\theta))(U \otimes R) H(x(\theta)) d \theta d s \leqslant \bar{\pi} \int_{\underline{\tau}_{2}}^{\bar{\tau}_{2}} \int_{t-s}^{t} H^{T}(x(\theta))(U \otimes R) H(x(\theta)) d \theta d s .
$$

Substituting (17)-(23) into (16) yields that

$$
\begin{aligned}
\mathscr{L} V\left(x_{t}, t, i\right)= & 2 x^{T}(t)\left(U \otimes P_{i}\right)\left(F(x(t), i)+G\left(x\left(t-\tau_{1, i}\right)\right)+\int_{t-\tau_{2, i}}^{t} H(x(s)) d s+\left(W \otimes \Gamma_{i}\right) x(t)\right) \\
& +\sum_{j=1}^{m} \pi_{i j} x^{T}(t)\left(U \otimes P_{j}\right) x(t)+\varepsilon_{0} \tau x^{T}(t)\left(U \otimes I_{N}\right) x(t)+\left(1+\bar{\pi}\left(\bar{\tau}_{1}-\underline{\tau}_{1}\right)\right) G^{T}(x(t))(U \otimes Q) G(x(t)) \\
& -G^{T}\left(x\left(t-\tau_{1, i}\right)\right)(U \otimes Q) G\left(x\left(t-\tau_{1, i}\right)\right)+\left(\tau_{2, i}+\frac{1}{2} \bar{\pi}\left(\bar{\tau}_{2}^{2}-\underline{\tau}_{2}^{2}\right)\right) H^{T}(x(t))(U \otimes R) H(x(t)) \\
& -\int_{t-\tau_{2, i}}^{t} H^{T}(x(s))(U \otimes R) H(x(s)) d s-\varepsilon_{0} \int_{t-\tau}^{t} x^{T}(s)\left(U \otimes I_{N}\right) x(s) d s .
\end{aligned}
$$

Noting that $U W=N W$, we have

$$
\left(U \otimes P_{i}\right)\left(W \otimes \Gamma_{i}\right)=(U W) \otimes\left(P_{i} \Gamma_{i}\right)=(N W) \otimes\left(P_{i} \Gamma_{i}\right) .
$$

With Lemma 1 and (25), we can deduce from (24) that

$$
\begin{aligned}
& \mathcal{L} V\left(x_{t}, t, i\right) \\
& \quad=\sum_{1 \leqslant k<l \leqslant N}\left[2 ( x _ { k } ( t ) - x _ { l } ( t ) ) ^ { T } \left(P_{i}\left(f\left(x_{k}(t), i\right)-f\left(x_{l}(t), i\right)\right)+P_{i}\left(g\left(x_{k}\left(t-\tau_{1, i}\right)\right)-g\left(x_{l}\left(t-\tau_{1, i}\right)\right)\right)\right.\right.
\end{aligned}
$$




$$
\begin{aligned}
& \left.+P_{i} \int_{t-\tau_{2, i}}^{t}\left(h\left(x_{k}(s)\right)-h\left(x_{l}(s)\right)\right) d s-N w_{k l} P_{i} \Gamma_{i}\left(x_{k}(t)-x_{l}(t)\right)\right)+\left(x_{k}(t)-x_{l}(t)\right)^{T} \bar{P}_{i}\left(x_{k}(t)-x_{l}(t)\right) \\
& +\left(g\left(x_{k}(t)\right)-g\left(x_{l}(t)\right)\right)^{T} c_{1} Q\left(g\left(x_{k}(t)\right)-g\left(x_{l}(t)\right)\right)+\left(h\left(x_{k}(t)\right)-h\left(x_{l}(t)\right)\right)^{T} c_{2 i} R\left(h\left(x_{k}(t)\right)-h\left(x_{l}(t)\right)\right) \\
& -\int_{t-\tau_{2, i}}^{t}\left(h\left(x_{k}(s)\right)-h\left(x_{l}(s)\right)\right)^{T} R\left(h\left(x_{k}(s)\right)-h\left(x_{l}(s)\right)\right) d s+\varepsilon_{0} \tau\left(x_{k}(t)-x_{l}(t)\right)^{T}\left(x_{k}(t)-x_{l}(t)\right) \\
& -\left(g\left(x_{k}\left(t-\tau_{1, i}\right)\right)-g\left(x_{l}\left(t-\tau_{1, i}\right)\right)\right)^{T} Q\left(g\left(x_{k}\left(t-\tau_{1, i}\right)\right)-g\left(x_{l}\left(t-\tau_{1, i}\right)\right)\right) \\
& \left.-\varepsilon_{0} \int_{t-\tau}^{t}\left(x_{k}(s)-x_{l}(s)\right)^{T}\left(x_{k}(s)-x_{l}(s)\right) d s\right]
\end{aligned}
$$

From Lemma 2, it follows that

$$
\begin{aligned}
& -\int_{t-\tau_{2, i}}^{t}\left(h\left(x_{k}(s)\right)-h\left(x_{l}(s)\right)\right)^{T} R\left(h\left(x_{k}(s)\right)-h\left(x_{l}(s)\right)\right) d s \\
& \quad \leqslant-\frac{1}{\tau_{2, i}}\left[\int_{t-\tau_{2, i}}^{t}\left(h\left(x_{k}(s)\right)-h\left(x_{l}(s)\right)\right)^{T} d s\right] R\left[\int_{t-\tau_{2, i}}^{t}\left(h\left(x_{k}(s)\right)-h\left(x_{l}(s)\right)\right) d s\right] .
\end{aligned}
$$

With the relationships (26) and (27), we have

$$
\mathcal{L} V\left(x_{t}, t, i\right) \leqslant \sum_{1 \leqslant k<l \leqslant N}\left[\xi_{k, l, i}^{T}(t) \Omega_{1}(k, l, i) \xi_{k, l, i}(t)-\varepsilon_{0} \int_{t-\tau}^{t}\left(x_{k}(s)-x_{l}(s)\right)^{T}\left(x_{k}(s)-x_{l}(s)\right) d s\right],
$$

where

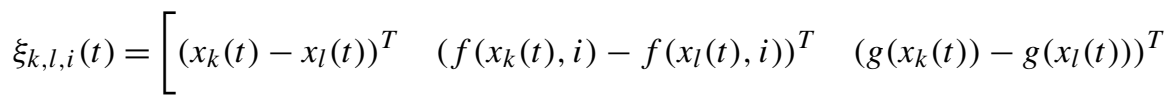

$$
\begin{aligned}
& \left.\left(g\left(x_{k}\left(t-\tau_{1, i}\right)\right)-g\left(x_{l}\left(t-\tau_{1, i}\right)\right)\right)^{T} \quad\left(h\left(x_{k}(t)\right)-h\left(x_{l}(t)\right)\right)^{T} \int_{t-\tau_{2, i}}^{t}\left(h\left(x_{k}(s)\right)-h\left(x_{l}(s)\right)\right)^{T} d s\right]^{T}, \\
& \Omega_{1}(k, l, i)=\left[\begin{array}{cccccc}
-N w_{k l} \hat{\Gamma}_{i}+\bar{P}_{i}+\varepsilon_{0} \tau I_{n} & P_{i} & 0 & P_{i} & 0 & P_{i} \\
* & 0 & 0 & 0 & 0 & 0 \\
* & * & c_{1} Q & 0 & 0 & 0 \\
* & * & * & -Q & 0 & 0 \\
* & * & * & * & c_{2 i} R & 0 \\
* & * & * & * & * & -\frac{1}{\tau_{2, i}} R
\end{array}\right]
\end{aligned}
$$

with $\hat{\Gamma}_{i}$ being defined in (13).

Notice that (4)-(6) implies

$$
\begin{aligned}
& {\left[\begin{array}{c}
x_{k}(t)-x_{l}(t) \\
f\left(x_{k}(t), i\right)-f\left(x_{l}(t), i\right)
\end{array}\right]^{T}\left[\begin{array}{cc}
\breve{B}_{1, i} & -\breve{B}_{2, i} \\
-\breve{B}_{2, i}^{T} & I
\end{array}\right]\left[\begin{array}{c}
x_{k}(t)-x_{l}(t) \\
f\left(x_{k}(t), i\right)-f\left(x_{l}(t), i\right)
\end{array}\right] \leqslant 0,} \\
& {\left[\begin{array}{c}
x_{k}(t)-x_{l}(t) \\
g\left(x_{k}(t)\right)-g\left(x_{l}(t)\right)
\end{array}\right]^{T}\left[\begin{array}{cc}
\breve{D}_{1} & -\breve{D}_{2} \\
-\breve{D}_{2}^{T} & I
\end{array}\right]\left[\begin{array}{c}
x_{k}(t)-x_{l}(t) \\
g\left(x_{k}(t)\right)-g\left(x_{l}(t)\right)
\end{array}\right] \leqslant 0,} \\
& {\left[\begin{array}{c}
x_{k}(t)-x_{l}(t) \\
h\left(x_{k}(t)\right)-h\left(x_{l}(t)\right)
\end{array}\right]^{T}\left[\begin{array}{cc}
\breve{V}_{1, i} & -\breve{V}_{2, i} \\
-\breve{V}_{2, i}^{T} & I
\end{array}\right]\left[\begin{array}{c}
x_{k}(t)-x_{l}(t) \\
h\left(x_{k}(t)\right)-h\left(x_{l}(t)\right)
\end{array}\right] \leqslant 0,}
\end{aligned}
$$

where $\breve{B}_{1, i}, \breve{B}_{2, i}, \breve{D}_{1}, \breve{D}_{2}, \breve{V}_{1}, \breve{V}_{2}$ are defined in (9)-(11). Then, from (28) and (31)-(33), we can infer that 


$$
\begin{aligned}
\mathcal{L} V\left(x_{t}, t, i\right) \leqslant & \sum_{1 \leqslant k<l \leqslant N}\left\{\xi_{k, l, i}^{T}(t) \Omega_{1}(k, l, i) \xi_{k, l, i}(t)-\varepsilon_{0} \int_{t-\tau}^{t}\left(x_{k}(s)-x_{l}(s)\right)^{T}\left(x_{k}(s)-x_{l}(s)\right) d s\right. \\
& +\left[\begin{array}{c}
x_{k}(t)-x_{l}(t) \\
f\left(x_{k}(t), i\right)-f\left(x_{l}(t), i\right)
\end{array}\right]^{T}\left[\begin{array}{cc}
-\delta_{1} \breve{B}_{1, i} & \delta_{1} \breve{B}_{2, i} \\
\delta_{1} \breve{B}_{2, i} & -\delta_{1} I
\end{array}\right]\left[\begin{array}{c}
x_{k}(t)-x_{l}(t) \\
f\left(x_{k}(t), i\right)-f\left(x_{l}(t), i\right)
\end{array}\right] \\
& +\left[\begin{array}{c}
x_{k}(t)-x_{l}(t) \\
g\left(x_{k}(t)\right)-g\left(x_{l}(t)\right)
\end{array}\right]^{T}\left[\begin{array}{cc}
-\delta_{2} \breve{D}_{1} & \delta_{2} \breve{D}_{2} \\
\delta_{2} \breve{D}_{2} & -\delta_{2} I
\end{array}\right]\left[\begin{array}{c}
x_{k}(t)-x_{l}(t) \\
g\left(x_{k}(t)\right)-g\left(x_{l}(t)\right)
\end{array}\right] \\
& \left.+\left[\begin{array}{c}
x_{k}(t)-x_{l}(t) \\
h\left(x_{k}(t)\right)-h\left(x_{l}(t)\right)
\end{array}\right]^{T}\left[\begin{array}{cc}
-\delta_{3} \breve{V}_{1} & \delta_{3} \breve{V}_{2} \\
\delta_{3} \breve{V}_{2} & -\delta_{3} I
\end{array}\right]\left[\begin{array}{c}
x_{k}(t)-x_{l}(t) \\
h\left(x_{k}(t)\right)-h\left(x_{l}(t)\right)
\end{array}\right]\right\} \\
= & \sum_{1 \leqslant k<l \leqslant N}\left[\begin{array}{c}
t \\
\xi_{k, l, i}^{T}(t) \Omega_{\vartheta}(k, l, i) \xi_{k, l, i}(t)-\varepsilon_{0} \int_{t-\tau}^{t}\left(x_{k}(s)-x_{l}(s)\right)^{T}\left(x_{k}(s)-x_{l}(s)\right) d s
\end{array}\right],
\end{aligned}
$$

where $\Omega_{\vartheta}(k, l, i)$ is defined in (14).

We are now ready to deal with the exponential synchronization of the network (1) or (2). For this purpose, we consider the following functional

$$
\mathcal{V}(x(t), t, r(t))=e^{\varepsilon t} V(x(t), t, r(t)),
$$

where $\varepsilon>0$ is a constant to be determined. Obviously, we can see that

$$
\mathcal{L} \mathcal{V}(x(t), t, i)=e^{\varepsilon t} \mathcal{L} V(x(t), t, i)+\varepsilon e^{\varepsilon t} V(x(t), t, i) .
$$

From (5) and (6), it can be inferred that there exists a constant $\alpha>0$ such that

$$
|g(x)-g(y)| \leqslant \alpha|x-y|, \quad \text { and } \quad|h(x)-h(y)| \leqslant \alpha|x-y|, \quad \forall x, y \in \mathbb{R}^{n} .
$$

Set

$$
c_{2}=\max _{1 \leqslant i \leqslant m}\left\{c_{2 i}\right\}, \quad \lambda_{0}=\max _{1 \leqslant i \leqslant m}\left\{\lambda_{\max }\left(P_{i}\right)\right\}, \quad c_{0}=c_{1} \lambda_{\max }(Q) \alpha^{2}+c_{2} \lambda_{\max }(R) \alpha^{2}+\varepsilon_{0} \tau .
$$

Then, for $V(x(t), t, i)$, we have

$$
\begin{aligned}
& V(x(t), t, i) \\
& \leqslant x^{T}(t)\left(U \otimes P_{i}\right) x(t)+\int_{t-\tau}^{t} G^{T}(x(s))(U \otimes Q) G(x(s)) d s \\
& \quad+\bar{\pi} \int_{\underline{\tau}_{1}}^{\bar{\tau}_{1}} \int_{t-\tau}^{t} G^{T}(x(\theta))(U \otimes Q) G(x(\theta)) d \theta d s+\int_{0}^{\tau_{2, i}} \int_{t-\tau}^{t} H^{T}(x(\theta))(U \otimes R) H(x(\theta)) d \theta d s \\
& \quad+\bar{\pi} \int_{\underline{\tau}_{2}}^{\bar{\tau}_{2}} \int_{0}^{u} \int_{t-\tau}^{t} H^{T}(x(\theta))(U \otimes R) H(x(\theta)) d \theta d s d u+\varepsilon_{0} \int_{0}^{\tau} \int_{t-\tau}^{t} x^{T}(\theta)\left(U \otimes I_{n}\right) x(\theta) d \theta d s \\
& =x^{T}(t)\left(U \otimes P_{i}\right) x(t)+c_{1} \int_{t-\tau}^{t} G^{T}(x(s))(U \otimes Q) G(x(s)) d s \\
& \quad+c_{2 i} \int_{t-\tau}^{t} H^{T}(x(s))(U \otimes R) H(x(s)) d s+\varepsilon_{0} \tau \int_{t-\tau}^{t} x^{T}(s)\left(U \otimes I_{n}\right) x(s) d s \\
& =\sum_{1 \leqslant k<l \leqslant N}\left[\left(x_{k}(t)-x_{l}(t)\right)^{T} P_{i}\left(x_{k}(t)-x_{l}(t)\right)+c_{1} \int_{t-\tau}^{t}\left(g\left(x_{k}(s)\right)-g\left(x_{l}(s)\right)\right)^{T} Q\left(g\left(x_{k}(s)\right)-g\left(x_{l}(s)\right)\right) d s\right. \\
& \left.\quad+c_{2 i} \int_{t-\tau}^{t}\left(h\left(x_{k}(s)\right)-h\left(x_{l}(s)\right)\right)^{T} R\left(h\left(x_{k}(s)\right)-h\left(x_{l}(s)\right)\right) d s+\varepsilon_{0} \tau \int_{t-\tau}^{t}\left(x_{k}(s)-x_{l}(s)\right)^{T}\left(x_{k}(s)-x_{l}(s)\right) d s\right]
\end{aligned}
$$




$$
\begin{aligned}
& \leqslant \sum_{1 \leqslant k<l \leqslant N}\left[\lambda_{\max }\left(P_{i}\right)\left|x_{k}(t)-x_{l}(t)\right|^{2}+c_{1} \lambda_{\max }(Q) \int_{t-\tau}^{t}\left|g\left(x_{k}(s)\right)-g\left(x_{l}(s)\right)\right|^{2} d s\right. \\
& \left.\quad+c_{2 i} \lambda_{\max }(R) \int_{t-\tau}^{t}\left|h\left(x_{k}(s)\right)-h\left(x_{l}(s)\right)\right|^{2} d s+\varepsilon_{0} \tau \int_{t-\tau}^{t}\left|x_{k}(s)-x_{l}(s)\right|^{2} d s\right] \\
& \leqslant \sum_{1 \leqslant k<l \leqslant N}\left[\lambda_{0}\left|x_{k}(t)-x_{l}(t)\right|^{2}+c_{0} \int_{t-\tau}^{t}\left|x_{k}(s)-x_{l}(s)\right|^{2} d s\right] .
\end{aligned}
$$

Now, choose $\varepsilon$ such that the following inequalities holds:

$$
\varepsilon \lambda_{0}+\lambda_{\max }\left(\Omega_{\vartheta}(k, l, i)\right) \leqslant 0 \quad(1 \leqslant k<l \leqslant N, 1 \leqslant i \leqslant m), \quad \varepsilon c_{0}-\varepsilon_{0} \leqslant 0 .
$$

Then, it follows readily from (34), (36) and (37) that

$$
\mathcal{L} \mathcal{V}(x(t), t, i) \leqslant 0 .
$$

By the generalized Itô formula (cf. [32,33]), we have

$$
\mathbb{E} \mathcal{V}(x(t), t, r(t))=\mathbb{E} \mathcal{V}(x(0), 0, r(0))+\mathbb{E} \int_{0}^{t} \mathcal{L} \mathcal{V}(x(s), s, r(s)) d s \leqslant \mathbb{E} \mathcal{V}(x(0), 0, r(0))
$$

which implies that

$$
e^{\varepsilon t} \mathbb{E} V(x(t), t, r(t)) \leqslant \mathbb{E} V(x(0), 0, r(0)) .
$$

Furthermore, it follows from (37) that

$$
\mathbb{E} V(x(0), 0, r(0)) \leqslant \mu_{0},
$$

where

$$
\mu_{0}=\sum_{1 \leqslant k<l \leqslant N}\left[\lambda_{0} \mathbb{E}\left\{\left|x_{k}(0)-x_{l}(0)\right|^{2}\right\}+c_{0} \mathbb{E} \int_{-\tau}^{0}\left|x_{k}(s)-x_{l}(s)\right|^{2} d s\right] .
$$

Letting $\rho=\min _{1 \leqslant i \leqslant m} \lambda_{\min }\left(P_{i}\right)$, we have

$$
\begin{aligned}
\mathbb{E} V(x(t), t, r(t)) & \geqslant \mathbb{E}\left\{x^{T}(t)\left(U \otimes P_{i}\right) x(t)\right\}=\sum_{1 \leqslant k<l \leqslant N} \mathbb{E}\left\{\left(x_{k}(t)-x_{l}(t)\right)^{T} P_{i}\left(x_{k}(t)-x_{l}(t)\right)\right\} \\
& \geqslant \rho \sum_{1 \leqslant k<l \leqslant N} \mathbb{E}\left\{\left|x_{k}(t)-x_{l}(t)\right|^{2}\right\} .
\end{aligned}
$$

This, together with (39) and (40), implies that

$$
\sum_{1 \leqslant k<l \leqslant N} \mathbb{E}\left\{\left|x_{k}(t)-x_{l}(t)\right|^{2}\right\} \leqslant \frac{\mu_{0}}{\rho} e^{-\varepsilon t},
$$

and therefore the network (1) or (2) is exponentially synchronized in the mean square.

Remark 3. In Theorem 1, the criteria are established that ensures that the coupled Markovian jumping complex network with mixed delays is globally synchronized in the mean square for all admissible parameter jumps and mode-dependent time-delays, and the criteria are expressed in terms of the solution to a set of LMIs. Note that LMIs can be effectively solved and checked by the algorithms such as the interior-point method from Matlab toolbox.

In order to show the generality of the main results obtained in Theorem 1, we consider two special cases for the complex network (1) or (2).

Case 1. In the network (1) or (2), let us assume that the network evolves at one mode only. In other words, there is no Markovian mode jumping. To be precise, in Eq. (2), let $F(x(t), r(t)), \tau_{1,(r)}, \tau_{2,(r)}$ and $\Gamma_{r(t)}$ be replaced by $F(x(t)), \tau_{1}, \tau_{2}$ and $\Gamma$, respectively. 
Then, Eq. (2) can be changed to

$$
\frac{d x(t)}{d t}=F(x(t))+G\left(x\left(t-\tau_{1}\right)\right)+\int_{t-\tau_{2}}^{t} H(x(s)) d s+(W \otimes \Gamma) x(t) .
$$

Furthermore, in Assumption 2, the condition (4) is replaced by

$$
\left[f(x)-f(y)-B_{1}(x-y)\right]^{T}\left[f(x)-f(y)-B_{2}(x-y)\right] \leqslant 0,
$$

where $B_{1}$ and $B_{2}$ are known constant matrices.

For the synchronization problem of the network (42), we have following results without proof.

Corollary 1. Under Assumptions 1 and 2 except with the condition (4) replaced by (43), the complex network (42) is globally exponentially synchronized if there exist three positive definite matrices $P>0, Q>0$ and $R>0$, and three positive scalar constants $\delta_{1}, \delta_{2}$ and $\delta_{3}$ such that the following LMIs hold:

$$
\Phi(k, l)=\left[\begin{array}{cccccc}
-N w_{k l}(P \Gamma+\Gamma P)+\Xi & P+\delta_{1} \breve{B}_{2} & \delta_{2} \breve{D}_{2} & P & \delta_{3} \breve{V}_{2} & P \\
* & -\delta_{1} I & 0 & 0 & 0 & 0 \\
* & * & Q-\delta_{2} I & 0 & 0 & 0 \\
* & * & * & -Q & 0 & 0 \\
* & * & * & * & \tau_{2} R-\delta_{3} I & 0 \\
* & * & * & * & * & -\frac{1}{\tau_{2}} R
\end{array}\right]<0
$$

$1 \leqslant k<l \leqslant N$

where $\breve{B}_{1}=\left(B_{1}^{T} B_{2}+B_{2}^{T} B_{1}\right) / 2, \breve{B}_{2}=\left(B_{1}^{T}+B_{2}^{T}\right) / 2, \Xi=-\delta_{1} \breve{B}_{1}-\delta_{2} \breve{D}_{1}-\delta_{3} \breve{V}_{1}$, and $\breve{D}_{1}, \breve{D}_{2}$, $\breve{V}_{1}$ and $\breve{V}_{2}$ are defined as in $(10)$ and (11).

Case 2. In this case, we consider the same array of complex networks with discrete time-delay only. Then, the complex network (1) reduces to

$$
\frac{d x(t)}{d t}=F(x(t), r(t))+G\left(x\left(t-\tau_{1, r(t)}\right)\right)+\left(W \otimes \Gamma_{r(t)}\right) x(t) .
$$

For the network (44), the following result follows readily.

Corollary 2. Under Assumptions 1 and 2, the network (44) is globally exponentially synchronized in the mean square if there exists a positive definite matrices $Q>0$, a set of positive definite matrices $P_{i}(i=1,2, \ldots, m)$, and two positive scalar constants $\delta_{1}$ and $\delta_{2}$ such that the following LMIs hold:

$$
\begin{gathered}
\Psi(k, l, i)=\left[\begin{array}{cccc}
-N w_{k l}\left(P_{i} \Gamma_{i}+\Gamma_{i} P_{i}\right)+\bar{P}_{i}+\Theta_{i} & P_{i}+\delta_{1} \breve{B}_{2, i} & \delta_{2} \breve{D}_{2} & P_{i} \\
* & -\delta_{1} I & 0 & 0 \\
* & * & c_{1} Q-\delta_{2} I & 0 \\
* & * & * & -Q
\end{array}\right]<0, \\
1 \leqslant k<l \leqslant N, \quad 1 \leqslant i \leqslant m,
\end{gathered}
$$

where $\Theta_{i}=-\delta_{1} \breve{B}_{1, i}-\delta_{2} \breve{D}_{1}$, and $\breve{B}_{1, i}, \breve{B}_{2, i}, \breve{D}_{1}, \breve{D}_{2}, \bar{P}_{i}$ and $c_{1}$ are defined as in Theorem 1 .

Remark 4. In this Letter, the complex network consists of $m$ modes and the network switches from one mode to another according to a Markovian chain with known transition probability. In our future work, we could further consider two independent Markovian jumping modes for node switching as well as network topology switching, which would reflect more features of the complexity. On the other hand, many networks in real world are directed and the study about directed dynamical networks is therefore quite interesting and useful. One of our future research topics would be the extension of our results to directed dynamical networks by referring to the techniques used in [34,35].

\section{A numerical example}

In this section, we present a simulation example so as to illustrate the usefulness of our main results. 
Phase Diagram of Mode 1

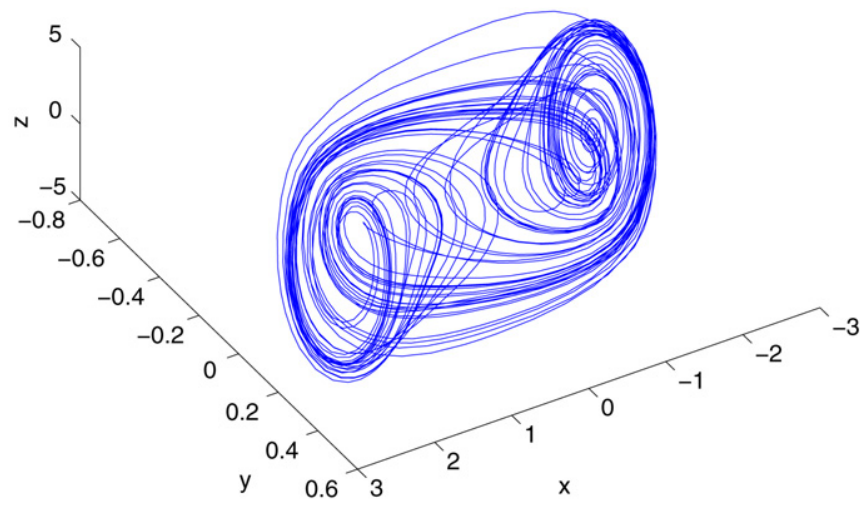

Phase Diagram of Mode 2

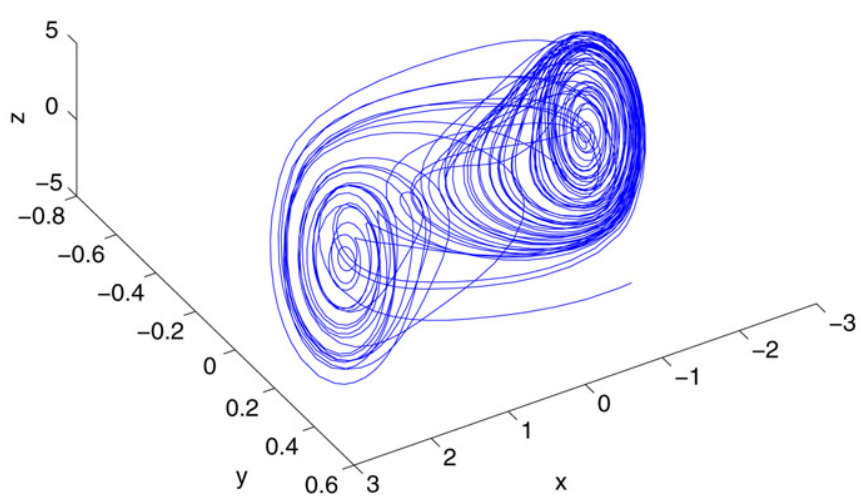

Fig. 1. Phase diagrams of the two modes.

For the sake of simplification, we consider a 3-node network with Markovian switching between two modes. The uncoupled dynamical subsystem of the $k$ th node $(k=1,2,3)$ in the network is given by

$$
\begin{aligned}
& \dot{x}_{k 1}=\alpha_{r(t)}\left(x_{k 2}-x_{k 1}-\sigma\left(x_{k 1}\right)\right)+\int_{t-\tau_{2, r(t)}}^{t} \tanh \left(0.6 x_{k 1}(s)\right) d s, \\
& \dot{x}_{k 2}=x_{k 1}-x_{k 2}+x_{k 3}+\int_{t-\tau_{2, r(t)}}^{t} 0.2 \cos \left(2 x_{k 2}(s)\right) d s, \\
& \dot{x}_{k 3}=-25.58 x_{k 2}-0.8 \sin \left(2 x_{k 1}\left(t-\tau_{1, r(t)}\right)\right)+\int_{t-\tau_{2, r(t)}}^{t} 0.5 \tanh \left(0.6 x_{k 3}(s)\right) d s,
\end{aligned}
$$

where

$$
\begin{aligned}
& \alpha_{1}=15, \quad \alpha_{2}=14, \\
& \tau_{1,1}=4, \quad \tau_{1,2}=5, \\
& \tau_{2,1}=0.5, \quad \tau_{2,2}=0.4, \\
& \sigma(s)=b s+\frac{1}{2}(a-b)(|s+1|-|s-1|)
\end{aligned}
$$

with $a=-5 / 7$ and $b=-8 / 7$. The phase portraits of system (45) with $r(t)=1$ and $r(t)=2$ are given in Fig. 1 .

Remark 5. System (45) without time delay terms is just the well-known Chua's circuit which possesses a chaotic attractor [36]. The dynamics analysis problems for system (45) with discrete time-delay but without distributed time-delay terms has been investigated in the literature, see, e.g., [37,38].

Now let us turn to the coupled complex network (1) or (2). Besides the parameters specified already, we further take

$$
\Pi=\left[\begin{array}{cc}
-3 & 3 \\
4 & -4
\end{array}\right], \quad W=\left[\begin{array}{ccc}
-2 & 1 & 1 \\
1 & -2 & 1 \\
1 & 1 & -2
\end{array}\right] \quad \Gamma_{1}=\operatorname{diag}\{15,15,15\}, \quad \Gamma_{2}=\operatorname{diag}\{10,10,10\} .
$$

Obviously, in (45), we have

$$
\begin{aligned}
& f\left(x_{k}, r(t)\right)=\left[\alpha_{r(t)}\left(x_{k 2}-x_{k 1}-\sigma\left(x_{k 1}\right)\right), x_{k 1}-x_{k 2}+x_{k 3},-25.58 x_{k 2}\right]^{T}, \\
& g\left(x_{k}\right)=\left[0,0,-0.8 \sin \left(2 x_{k 1}\right)\right]^{T}, \\
& h\left(x_{k}\right)=\left[\tanh \left(0.6 x_{k 1}\right), 0.2 \cos \left(2 x_{k 2}\right), 0.5 \tanh \left(0.6 x_{k 3}\right)\right]^{T} .
\end{aligned}
$$



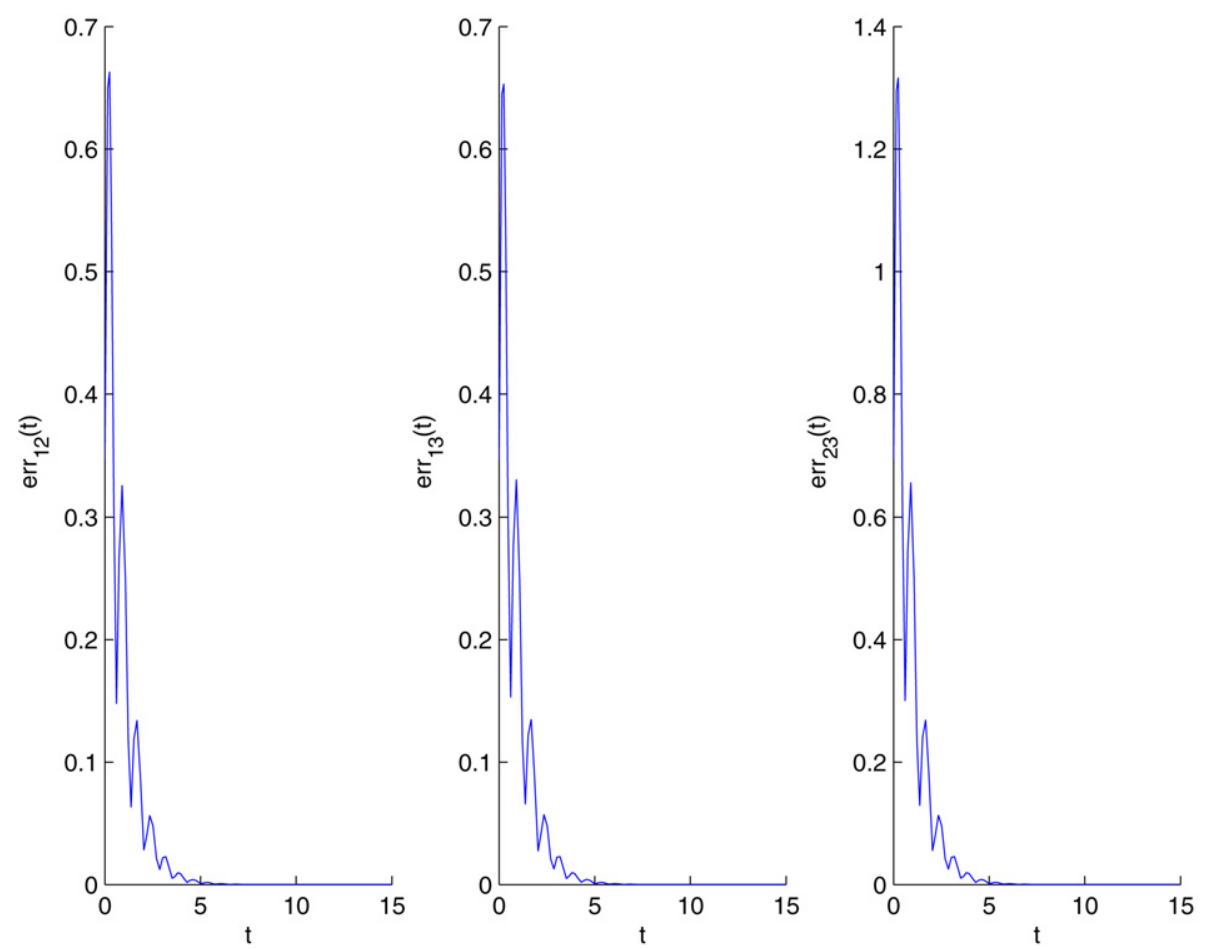

Fig. 2. State trajectory of the synchronization error.

With the parameters given above, it can be verified that

$$
\begin{aligned}
& B_{1,1}=\left[\begin{array}{ccc}
-15(1-8 / 7) & 15 & 0 \\
1 & -1 & 1 \\
0 & -25.58 & 0
\end{array}\right], \quad B_{2,1}=\left[\begin{array}{ccc}
-15(1-5 / 7) & 15 & 0 \\
1 & -1 & 1 \\
0 & -25.58 & 0
\end{array}\right], \\
& B_{1,2}=\left[\begin{array}{ccc}
-14(1-8 / 7) & 15 & 0 \\
1 & -1 & 1 \\
0 & -25.58 & 0
\end{array}\right], \quad B_{2,2}=\left[\begin{array}{ccc}
-14(1-5 / 7) & 15 & 0 \\
1 & -1 & 1 \\
0 & -25.58 & 0
\end{array}\right], \\
& D_{1}=\operatorname{diag}\{1.6,0,0\}, \quad D_{2}=\operatorname{diag}\{-1.6,0,0\}, \quad V_{1}=\operatorname{diag}\{0.6,0.4,0.3\}, \\
& \bar{\tau}_{1}=5, \quad \underline{\tau}_{1}=4, \quad \bar{\tau}_{2}=0.5, \quad \underline{\tau} 2=0.4, \quad \tau=5, \quad \bar{\pi}=4 .
\end{aligned}
$$

By using the Matlab LMI toolbox, we solve the LMI (8) and obtain the feasible solutions as follows:

$$
\begin{aligned}
& P_{1}=\left[\begin{array}{ccc}
0.1819 & -0.0198 & 0.0360 \\
-0.0198 & 0.0746 & 0.0390 \\
0.0360 & 0.0390 & 0.1777
\end{array}\right], \quad P_{2}=\left[\begin{array}{ccc}
0.1428 & -0.0090 & 0.0490 \\
-0.0090 & 0.1581 & 0.0209 \\
0.0490 & 0.0209 & 0.1538
\end{array}\right], \\
& Q=\left[\begin{array}{lll}
0.0057 & 0.0000 & 0.0022 \\
0.0000 & 0.0066 & 0.0000 \\
0.0022 & 0.0000 & 0.0031
\end{array}\right], \quad R=\left[\begin{array}{ccc}
60.8122 & -0.0001 & 0.0912 \\
-0.0001 & 123.5552 & 0.0001 \\
0.0912 & 0.0001 & 115.9344
\end{array}\right] \text {, } \\
& \delta_{1}=0.0755, \quad \delta_{2}=0.0577, \quad \delta_{3}=186.6805 \text {. }
\end{aligned}
$$

Therefore, it follows from Theorem 1 that the network (1) or (2) with given parameters is globally exponentially synchronized in the mean square.

To confirm the theoretical results via simulation, we let $\operatorname{err}_{k l}(t)=\left|x_{k}(t)-x_{l}(t)\right|$, and

$$
\left[x_{1}^{T}(s) \quad x_{2}^{T}(s) \quad x_{3}^{T}(s)\right]^{T}=[-1.6 ; 0 ; 1.6 ;-1.4 ;-0.2 ; 1.8 ;-1.8 ; 0.2 ; 1.4]^{T}, \quad-5 \leqslant s \leqslant 0 .
$$

Then, it can be seen from Fig. 2 that the synchronization errors between different nodes indeed approach zero, and our objective of synchronization is well achieved.

\section{Conclusions}

In this Letter, we have investigated the exponential synchronization problem for an array of linearly coupled complex networks with Markovian jumping and mixed time-delays. Both the discrete and distributed time-delays are dependent on network modes. By 
utilizing a novel Lyapunov-Krasovskii functional and the Kronecker product, we have shown that the addressed synchronization problem is solvable if a set of linear matrix inequalities (LMIs) are feasible. A unified LMI approach has been developed to establish sufficient conditions for the coupled complex networks to be globally exponentially synchronized in the mean square, and a simulation example has been provided to show the usefulness of the proposed global exponential synchronization condition.

\section{References}

[1] D.J. Watts, S.H. Strogatz, Nature 393 (1998) 440.

[2] X.-F. Wang, G. Chen, Int. J. Bifur. Chaos 12 (1) (2002) 187.

[3] J. Jost, M. Joy, Phys. Rev. E 65 (2002) 061201.

[4] J. Liang, Z. Wang, X. Liu, Exponential synchronization of stochastic delayed discrete-time complex networks, Nonlinear Dynam., in press.

[5] A. Arenas, A. Díaz-Guilera, C.J. Pérez-Vicente, Physica D: Nonlinear Phenomena 224 (1-2) (2006) 27.

[6] A. Zheleznyak, L.O. Chua, Int. J. Bifur. Chaos 4 (3) (1994) 639.

[7] V. Perez-Munuzuri, V. Perez-Villar, L.O. Chua, IEEE Trans. Circuits Syst.-I 40 (1993) 174.

[8] L.M. Pecora, T.L. Carroll, Phys. Rev. Lett. 64 (8) (1990) 821.

[9] Z. Li, G. Chen, IEEE Trans. Circuits Syst.-II 53 (1) (2006) 28.

[10] C.W. Wu, IEEE Trans. Circuits Syst.-II 52 (5) (2005) 282.

[11] H. Gao, T. Chen, IEEE Trans. Automat. Control 52 (2007) 328.

[12] H. Gao, J. Lam, G. Chen, Phys. Lett. A 360 (2006) 263.

[13] M.S. Mahmoud, Y. Shi, H.N. Nounou, Int. J. Innovative Comput. Inform. Control 3 (2) (2007) 407.

[14] M. Basin, E. Sanchez, R. Martinez-Zuniga, Int. J. Innovative Comput. Inform. Control 3 (5) (2007) 1309.

[15] C.G. Li, G. Chen, Physica A 343 (2004) 263.

[16] Z. Wang, Y. Liu, K. Fraser, X. Liu, Phys. Lett. A 354 (4) (2006) 288.

[17] Z. Wang, Y. Liu, M. Li, X. Liu, IEEE Trans. Neural Networks 17 (3) (2006) 814.

[18] W.L. Lu, T.P. Chen, IEEE Trans. Circuits Syst.-I 51 (12) (2004) 2491.

[19] W.L. Lu, T.P. Chen, G. Chen, Physica D: Nonlinear Phenomena 221 (2) (2006) 118.

[20] M.P. Casey, Neural Comput. 8 (6) (1996) 1135.

[21] P. Tino, M. Cernansky, L. Benuskova, IEEE Trans. Neural Networks 15 (1) (2004) 6.

[22] Z. Wang, Y. Liu, L. Yu, X. Liu, Phys. Lett. A 356 (4-5) (2006) 346.

[23] C. Busch, M. Magdon-Ismail, M. Mavronicolas, J. Parallel Distrib. Comput. 67 (2007) 1168.

[24] W.-M. Chen, C.-S. Li, F.-Y. Chiang, H.-C. Chao, Comput. Commun. 30 (2007) 2892.

[25] I. Gunawan, Reliability Eng. Syst. Safety 93 (2008) 271.

[26] J.J. Torres, J. Marro, P.L. Garrido, J.M. Cortes, F. Ramos, M.A. Munoz, Biophys. Chem. 115 (2005) 285.

[27] Y. Ji, H.J. Chizeck, IEEE Trans. Automat. Control 35 (1990) 777.

[28] P. Shi, Y. Xia, G. Liu, D. Rees, IEEE Trans. Automat. Control 51 (1) (2006) 97.

[29] L. Hu, P. Shi, B. Huang, J. Math. Anal. Appl. 313 (2) (2006) 504.

[30] Y. Liu, Z. Wang, X. Liu, Neural Networks 19 (5) (2006) 667.

[31] K.Q. Gu, An integral inequality in the stability problem of time-delay systems, in: Proceedings of 39th IEEE Conference on Decision and Control, December 2000, Sydney, Australia, 2000, pp. 2805-2810.

[32] R.Z. Khasminskii, Stochastic Stability of Differential Equations, Alphen aan den Rijn, Sijthoffand Noor, Khasminskiidhoff, 1980.

[33] A.V. Skorohod, Asymptotic Methods in the Theory of Stochastic Differential Equations, Amer. Math. Soc., Providence, RI, 1989.

[34] J.Q. Lu, D.W.C. Ho, Chaos Solitons Fractals 37 (2008) 1497.

[35] J.Q. Lu, D.W.C. Ho, M. Liu, Phys. Lett. A 369 (5-6) (2007) 444.

[36] L.O. Chua, C.W. Wu, A. Huang, G.-Q. Zhong, IEEE Trans. Circuits Syst.-I 40 (1993) 732.

[37] X.F. Wang, G.Q. Zhong, K.F. Tang, Z.F. Liu, IEEE Trans. Circuits Syst.-I 48 (9) (2001) 1151.

[38] C. Cruz-Hernández, N. Romero-Haros, Commun. Nonlinear Sci. Numer. Simul. 13 (2008) 645. 